\title{
Monastères et scriptoria en Lotharingie (IXe -Xe siècles)
}

Jens Schneider

\section{Citer ce document / Cite this document :}

Schneider Jens. Monastères et scriptoria en Lotharingie (IXe -Xe siècles). In: Bulletin de la Commission royale d'histoire. Académie royale de Belgique. Tome 176/2, 2010. pp. 21-39;

doi : https://doi.org/10.3406/bcrh.2010.1085

https://www.persee.fr/doc/bcrh_0001-415x_2010_num_176_2_1085

Fichier pdf généré le 19/03/2019 


\title{
Monastères et scriptoria en Lotharingie $\left(\mathrm{IX}^{\mathrm{e}}-\mathrm{X}^{\mathrm{e}} \text { siècles }\right)^{1}$
}

\author{
par Jens Schneider \\ Université de Limoges
}

\section{Introduction}

Un scriptorium est le lieu de travail de scribes. La notion est traditionnellement associée à un monastère mais cet atelier peut se situer également au sein d'un évêché ou dans l'entourage d'un comte. Cependant, l'existence de scriptoria épiscopaux, et plus encore de scriptoria comtaux, avant la mise en place de véritables chancelleries, reste parfois virtuelle pendant la période envisagée dans la présente contribution ${ }^{2}$. Si la société (ou plutôt les élites) christianisée du $\mathrm{Ix}^{\mathrm{e}}$ siècle a en quelque sorte abandonné le domaine de l'écrit au Nord des Alpes, «le laissant ici aux hommes d'Église » ${ }^{3}$, qui a alors rédigé les actes de la pratique? La place des notaires professionnels a été occupée rapidement par les monastères qui auraient monopolisé la production diplomatique ${ }^{4}$, à l'exception des chancelleries royales et impériales. Face à la «cléricalisation de la diplo-

I Je remercie vivement Laurent Morelle, EPHS Paris, d'avoir accepté de faire une relecture critique du présent article.

${ }^{2}$ Gh. Brunel, Chartes et chancelieries épiscopales dans le Nord de la France au $\mathrm{xI}^{\mathfrak{c}}$ siècle, dans M. Parisse dir., A propos des actes d'évêques, Nancy, 1991, p. 227-244 O. Guyotjeannin, J. Pycke, B.-M. Tock, Diplomatique médiévale, Turnhout, 1993 (L'Atelier du médiéviste), p. 114, 223-225 - O. GuYOTJEANNIN, Les sources de l'histoire médiévale, Paris, 1998, p. 176-178 - B.-M. Tock, L'acte privé en France, viI ${ }^{\mathrm{e}}$ siècle - milieu $\mathrm{x}^{\mathrm{e}}$ siècle, Mélanges de l'ÉCOle fRANÇAISE de Rome, 111 , 1999, p. 499-537 - F. Bougard, Mise en écriture et production documentaire en Occident, dans L'autorité de l'écrit au Moyen Age (Orient-Occident), Paris, 2009 (Histolre anCIENNE et Médiévale, 102), p. 13-20-Cf. la contribution de M. MARGUE dans le présent volume.

${ }^{3}$ Bougard, Mise en écriture, p. 17 - Cf. Tock, L'acte privé.

4 O. Guyotjeannin, "Penuria scriptorum». Le mythe de lanarchie documentaire dans la France du Nord ( $\mathrm{x}^{\mathrm{e}}$ - première moitié du $\mathrm{xI}^{\mathrm{e}}$ siècle), BiblıTHÈ̀ue DE L'ÉCOLE Des Chartes, 155, 1997, p. 11-44. Avec plus de réserve L. Morelle, Incertitudes et fauxsemblants: quelques remarques sur l'élaboration des actes privés carolingiens à la lumière de deux gisements de France septentrionale (Sithiu/Saint-Bertin, Saint-Denis), dans P. ERHART, K. Heidecker, B. Zeller dir., Die Privaturkunden der Karolingerzeit, Dietikon-Zürich, 2009, p. 103-120 - Au sujet des notaires professionnels voir G. DESPY, Les chartes privées comme sources de l'histoire rurale pendant les temps mérovingiens et carolingiens, dans 
matique ${ }^{5}$ il convient alors de s'interroger sur l'importance des différentes communautés religieuses chargées de la rédaction de chartes. La présente étude dresse d'abord un état des lieux des scriptoria monastiques dans l'espace lotharingien. Dans une deuxième partie sera présenté un corpus de quelque 260 actes privés. Sur cette base, on étudiera la répartition de la production diplomatique et son évolution au cours d'un siècle. En conclusion, on proposera quelques observations au sujet du rôle des fondations monastiques et des scriptoria au service des grands pour revenir au problème du lieu de la production de chartes, monastère ou autre.

Puisqu'il va être question de la production diplomatique dans les villes et campagnes, il convient de préciser l'espace considéré. Bien que focalisée sur "les anciens Pays-Bas» ${ }^{6}$, cette étude doit envisager d'abord la Lotharingie entière, ce qui me permet d'apporter quelques observations comparatives. Avec Michel Parisse, le terme «Lotharingie» sera utilisé ici pour désigner non pas la vaste Francia Media de Lothaire I $^{\text {er }}$ qui s'étendait de la Mer du Nord à la Méditerranée ${ }^{7}$, mais le regnum Lotharii créé en 855 pour l'arrière-petit-fils de Charlemagne ${ }^{8}$. Le royaume de Lothaire II comprenait dans les premiers temps des terres jurassiennes et rhodaniennes et suivait plus ou moins une sorte de colonne vertébrale Utrecht Liège - Trèves - Metz - Besançon ${ }^{9}$. Après de nombreux annexions et partages, il se présentait au $\mathrm{X}^{\mathrm{e}}$ siècle sans le Jura et avec une partie très restreinte de la Frise (voir la carte 1$)^{10}$.

C'est vers le milieu du $\mathrm{x}^{\mathrm{e}}$ siècle que se termine le premier siècle lotharingien. Les années 950/960, avec le couronnement impérial d'Otton $\mathrm{I}^{\text {er }}$ et le gouvernement de son frère Brunon de Cologne, chancelier, archevêque de Cologne et archidux ${ }^{11}$ en Lotharingie, semblent communément admises comme une césure. Il n'est pas surprenant que cet espace lotharingien

H. Atsma dir., La Neustrie. Les pays au Nord de la Loire de 650 à 850, Sigmaringen, 1989, p. 583-593; R. LE JAN, À la recherche des élites rurales du début du viII ${ }^{\mathrm{c}}$ siècle: le notaire alsacien Chrodoin, Revue du NORD, 86, 2004, p. 485-498.

5 Tock, L'acte privé, p. 535.

6 Pour l'invitation au colloque «Chancelleries princières et Scriptoria dans les anciens Pays-Bas, $\mathrm{x}^{\mathrm{e}}-\mathrm{Xv}^{\mathrm{e}}$ siècles» je tiens à remercier les organisateurs auprès de la Commission royale d'Histoire de Belgique ainsi que Michel Margue, Université du Luxembourg.

$7 \mathrm{Cf}$. les deux colloques multi-site tenus en 2006: «De la Mer du Nord à la Méditerranée: Francia media, une région au ccur de l'Europe (ca. 840-1050)», Metz, Luxembourg, Trèves; «Francia Media. Culture and Cultural Exchanges in the Heart of Europe (8501050)», Bruxelles, Gent, Oudenaarde.

8 S. KASCHKE, Die dispositio regni Lothars I. von 855, dans R. NOLDEN dir., Lothar $I$. Kaiser und Mönch in Prüm, Trèves, 2005, p. 89-98.

9 R. PARISOT, Le royaume de Lorraine sous les Carolingiens (843-923), Paris, 1898.

10 Sur les parties de la Frise revenues aux Lothaire père et fils voir K. VAN VLIET, In kringen van kanunniken. Munsters en kapittels in het bisdom Utrecht 695-1227, Zutphen, 2002, p. 133-138 - J. SCHNEIDER, Auf der Suche nach dem verlorenen Reich. Lotharingien im 9. und 10. Jahrhundert, Cologne, Weimar, Vienne (Publications du CLUDEM, 30), 2010, p. 78-91 et carte 10.2 , p. 646 .

11 Ruotger, Lebensbeschreibung des Erzbischofs Bruno von Köln, éd. I. OTT, Cologne, Graz, 1951 (MGH SRG N. S. 10), c. 20, p. 19. 
artificiellement créé connaisse un processus de dilatation dans la deuxième moitié du $\mathrm{x}^{\mathfrak{e}}$ siècle, après être devenu partie intégrante de la Francie orientale puis de l'Empire. L'année 959 ne peut certainement plus être considérée comme le moment de la création de deux duchés, haut- et bas-lotharingiens $^{12}$; toujours est-il que Flodoard de Reims mentionne à cette date la nomination à la dignité ducale de Frédéric par Brunon ${ }^{13}$. Pour les anciens Pays-Bas, Godefroid de Verdun suivra, ou bien est-il peut-être déjà en fonction ${ }^{14}$. On observe un processus de polarisation jusqu'au moment où, en 1033, le roi Conrad II réunit l'espace bourguignon-provençal à l'Empire. Du milieu du $\mathrm{x}^{\mathrm{e}}$ au milicu du $\mathrm{XI}^{\mathrm{e}}$ siècle, il y a eu plusieurs tentatives contre l'autorité ottonienne puis salienne ${ }^{15}$. Prenons comme exemple le roi carolingien du royaume occidental, Lothaire, qui va entreprendre en 978 une campagne éclair à Aix-la-Chapelle pour retourner l'Aigle qui surmontait le palais royal ${ }^{16}$. Cette anecdote célèbre montre deux choses: les futurs rois de France ont gardé la mémoire de leur héritage lotharingien, de cette hereditas largior, que Charles le Simple faisait mentionner dans ses diplômes à partir de $911^{17}$; en deuxième lieu on constate que l'espace lotharingien est devenu partie intégrante de l'Empire, sans que les rois de France ou d'ambitieux ducs de Lotharingie aient réussi à imposer leur autorité. Le petit-neveu de Frédéric, Gozélon, et son fils Godefroid le

12 R. BARTh, Der Herzog in Lotharingien im 10. Jahrhundert, Sigmaringen, 1990.

13 Les Annales de Flodoard, ed. Ph. LAuer, Paris, 1905 (Coll. DE TEXTES POUR SER VIR À L'ÉTUdE FT À L'INSEIGNEMENT DE L'HISTOIRE, 28), a. 959: ... Fredericum quendam comitem eis vice sua prefecit. Cf. Chronique et chartes de l'abbaye de Saint-Mihiel, éd. A. LESORT, Paris, 1909-1912 (Mettensia, 6), no 27 a. 962: Ego Fridericus Dei miserante gratia dux Lothariensium - Pour un changement dans la représentation du pouvoir H. KELLER, Das neue Bild des Herrschers. Zum Wandel der „Herrschaftsrepräsentation” unter Otto dem Großen, dans B. Schneidmüller, St. Weinfurter dir., Ottonische Neuanfänge, Mayence, 2001, p. 189-211.

14 Recueil des chartes de l'abbaye de Stavelot-Malmédy, éd. J. Halkin, Ch.-G. Roland, vol. 1, Bruxelles 1909 (CRH), no 74 [a. 958-964]: regnante ... Godefrido duce. MGH DD O.I, $\mathrm{n}^{\circ} 291$ a. 965 : Godefridus bone memoriae dux noster.

15 M. PARISSE, Désintégration et regroupements territoriaux dans les principautés lotharingiennes du XI ${ }^{e}$ au XIII ${ }^{\mathrm{e}}$ siècle, dans A. HeIt dir., Zwischen Gallia und Germania, Frankreich und Deutschland. Konstanz und Wandel raumbestimmender Kräfte, Trèves, 1987 (TRIERER Historische Forschungen, 12), p. 154-180 - IDEM, Les hommes et le pouvoir dans la Lorraine de l'an Mil, dans D. Iogna-PRAt, J.-Ch. PICARD dir., Religion et culture autour d' l'an Mil. Royaume capétien et Lotharingie, Paris, 1990 (Hugues Capet 987-1987. La France de l'an Mil), p. 259-266 - B. SCHNEIDmüller, Regnum und Ducatus. Identität und Integration in der lothringischen Geschichte des 9. bis 11. Jahrhunderts, RhinisChe Viertel.JAHRsBLÄtTer, 51, 1987, p. 81-114- K. F. Wlerner, Politische und kirchliche Konflikte in Lotharingien und Burgund im Spiegel des lateinischen Tierepos (10.-11. Jahrhundert), RHEINISCHE: VIERTELJAHRSBLÄTTER, 61, 1997, p. 1-33.

16 Richer de Saint-Remi, Historiae, éd. H. HoffmanN, Hanovre, 2000 (MGH SS 38), III.71. Cf. Werner, Konflikte, p. 9-10 - J. RöMER, Der Adler als Symbol Karls des Großen? Ein Blick in bisher unbeachtete Quellen, dans F.-R. ERKENs dir., Karl der Große und das Erbe der Kulturen, Berlin, 2001, p. 150-151.

17 Recueil des actes de Charles III le Simple, roi de France (893-923), éd. Ph. Lauer,

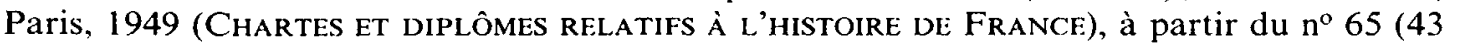
sur 123 diplômes). 
Barbu ont su réunir un temps les terres lotharingiennes mais cela n'a pas duré. On peut alors constater que c'est vers le milieu du $\mathrm{xI}^{\mathrm{e}}$ siècle qu'un duché de Basse-Lotharingie, au sein de l'Empire, représente une structure politique relativement stable.

\section{Les chartes dans l'espace lotharingien}

Dans le cadre d'une recherchc sur la cohérence de l'espace lotharingien, nous avons rassemblé un catalogue de toutes les chartes concernant la Lotharingie de l'année 855 jusqu'en $959^{18}$. Ces chartes ont été rédigées pour la plupart dans un scriptorium ecclésiastique, plus précisément un monastère ou une collégiale, sachant qu'il n'est pas toujours possible d'identifier le lieu d'émission. Le $\mathrm{xI}^{\mathrm{e}}$ siècle et ses chancelleries épiscopales de plus en plus professionnelles paraît loin ${ }^{19}$, et encore est-il parfois problématique, comme il a été démontré lors de ce colloque, d'attribuer une chancellerie à une cité épiscopale ${ }^{20}$. Le paysage des scriptoria est donc, avant l'an mil, essentiellement un paysage d'institutions de vita communis. L'expression de «paysage»n'est pas employée ici pour signifier une cohérence quelconque mais pour résumer la totalité des institutions dans cet espace politique lotharingien ${ }^{21}$.

La carte $1^{22}$ rend compte de la répartition spatiale des lieux de production. Les points noirs indiquent les monastères ou collégiales émetteurs de chartes: Saint-Sauveur d'Utrecht, Saint-Servais de Maastricht, SaintTrond, Brogne, Salles, Crespin et Saint-Ghislain, Stavelot; cn Rhćnanie Sainte-Ursule de Cologne, Saints-Cassius-et-Florent de Bonn, puis Prüm, Echternach, Saint-Maximin devant Trèves, Mettlach; en Lorraine Metz avec Saint-Arnoul mais aussi, avec une charte, Saint-Pierre-auxNonnains, puis Gorze, Bouxières, Saint-Vanne de Verdun, Saint-Mihiel, Saint-Evre de Toul, et enfin quatre chartes en Alsace, dont une rédigée au monastère de Munster (Münster im Gregoriental) et trois à Strasbourg. Il paraît légitime de considérer une bonne partie des chartes destinées

18 SCHNEIDER, Suche, annexe 4, p. 500-519.

19 Brunel, Chartes, p. 228-239 - Guyotjeannin, Pycke, Tock, Diplomatique, p. 114 - L. Morelle, Histoire et archives vers l'an mil: une nouvelle "mutation"?, Histoire ET ARCHIVES, 3, 1998, p. 119-141.

20 Une chancellerie épiscopale semble probable à Cambrai, moins probable à Thérouanne et inexistante à Liège: voir les contributions de B.-M. TOCK et J.-L. KuPPER dans le présent volume. Cf. Brunel, Chartes.

21 Sur le terme de paysage très en vogue cf. les réflexions de H.-D. HeimanN, J. SCHNEIDER, Kloster - Landschaft - Klosterlandschaft? Annäherungen und Ausblick, dans R. CzAJA, H.-D. HeimanN, M. Wemhoff dir., Klosterlandschaften. Methodisch-exemplarische Annäherungen, Munich, 2008 (MittelalterStudien, 16), p. 9-22 - J. Schneider, La Lotharingie était-elle une région historique? dans Construction de l'espace au Moyen Age: pratiques et représentations, Paris, 2007 (HistoIRe ANCIENNE ET MÉdiévale, 96), p. 425-433.

22 Je remercie Rémi Crouzevialle, Université de Limoges, pour sa collaboration cartographique. 


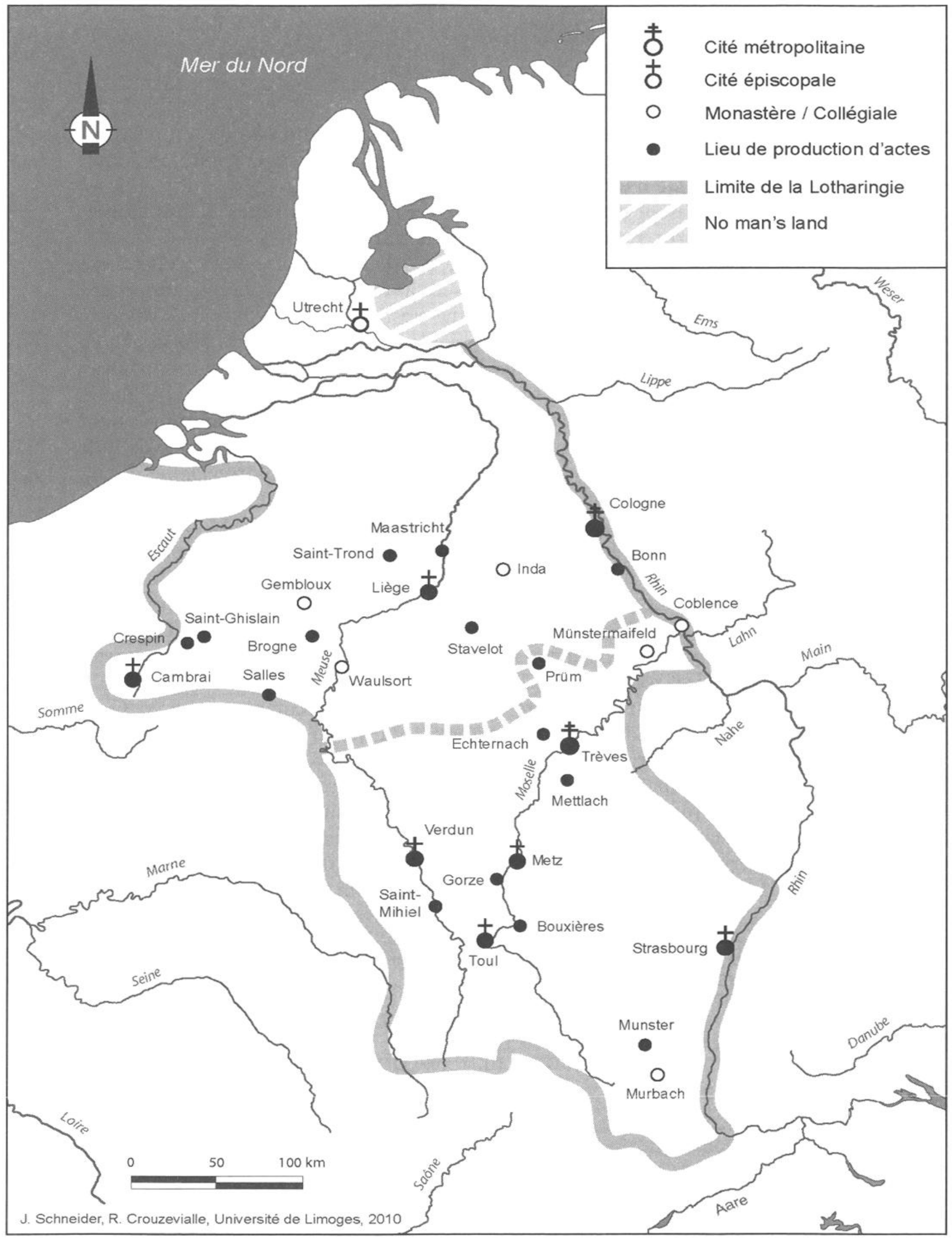

Carte 1: Lieux de production d'actes (855-959). 
à une communauté religieuse comme le produit d'un scribe monastique qui n'appartient pas forcément à la communauté bénéficiaire, en outre sa communauté n'est pas forcément non plus l'émettrice de l'acte. Il n'est alors pas suffisant d'étudier les lieux d'émanation des actes; il faut ajouter les destinataires, représentés sur la carte par un rond.

L'analyse de la carte donne lieu à plusieurs surprises. On y cherchera en vain quelques institutions supposées avoir joué un rôle plus important que d'autres, notamment en tant que destinataires de diplômes royaux: du nord au sud, on mentionnera Nivelles ${ }^{23}$, Chèvremont ${ }^{24}$, Hornbach en Palatinat $^{25}$ et Andlau en Alsace ${ }^{26}$. Remiremont, célèbre pour son Liber memorialis, ne figure pas non plus sur la carte. Cela veut dire qu'aucun acte ou diplôme conservé n'a été donné ou destiné à Remiremont pendant la période étudiée. On constate le même phénomène pour l'évêque de Bâle et son chapitre. Signalons enfin le cas particulier de Notre-Dame d'Aixla-Chapelle. La collégiale a reçu cinq diplômes pendant notre période mais elle ne semble pas avoir donné ou reçu aucune charte ${ }^{27}$. C'est néanmoins le seul exemple d'un palais mentionné comme lieu d'émission d'une charte. Il s'agit d'un acte d'échange confirmé par Brunon de Cologne un 31 octobre d'une année comprise entre 958 et 964 , et qui a peut-être été rédigé à Stavelot bien que la souscription ne mentionne pas de chancelier ou scribe, contrairement à ce que l'on constate dans d'autres chartes émanant de ce scriptorium ${ }^{28}$.

Ce premier examen montre une situation relativement équilibrée. C'est un constat qui étonne de prime abord, vu qu'on a toujours tendance à voir l'ancienne province de Belgique première (province ecclésiastique de Trèves sans la partie outre-Rhin) en avance par rapport à la partie septentrionale, la future Basse-Lotharingie. La situation s'explique en partie par l'état de la tradition: la plupart des actes conservés dans le Livre d'or d'Echternach ou les chartes de Bouxières ne nous renseignent pas sur le lieu de production ${ }^{29}$. Mais d'autres facteurs ont joué.

23 Recueil des actes de Charles II le Chauve, roi de France, éd. A. GiRY, M. Prou, G. Tessier, Paris, 1943-1955 (Chartes et diplômes relatifs Ȧ l'historre de France), $\mathrm{n}^{\circ}$ 433; MGH DD Zw, n 16, DD LK, n 50.

24 MGH DD LK, $n^{\circ} 18,70$, DD O.I, $\mathrm{n}^{\circ} 88$.

25 MGH DD Lo.II, n 24; Recueil Charles II, éd. GIRY, n 5; MGH DD O.I, nº 117.

26 MGH DD K.III, $\mathrm{n}^{\circ} 24,95,96$, DD LK, $\mathrm{n}^{\circ} 68$.

27 MGH DD LDt., $n^{\circ} 133$, DD K.III, n 109, DD Arn, n 31, DD H.I, nº 23, DD O.I, $\mathrm{n}^{\circ} 42$

28 Recueil Stavelot, éd. Halkin, Roland, $\mathrm{n}^{\circ} 74$; Urkunden- und Quellenbuch zur Geschichte der althuxemburgischen Territorien bis zur burgundischen Zeit, éd. C. WAMPACH, vol. 1 (bis zum Friedensvertrag von Dinant 1199), Luxembourg, 1935, ${ }^{\circ} 167$ : Acta sunt hec publice Aquis palatio ...

${ }^{29}$ C. WAMPaCH, Geschichte der Grundherrschaft Echternach im Frühmittelalter. Untersuchungen über die Person des Gründers, über die Kloster- und Wirtschaftsgeschichte auf Grund des liber aureus Epternacensis (698-1222), 2 vol., Luxembourg, 1929-1930; Les origines de l'abbaye de Bouxières-aux-Dames au diocèse de Toul. Reconstitution du chartrier et édition critiques des chartes antéricures à 1200, éd. R.-H. BAUTIER, Nancy, 1987 (RECUEIL DE DOCUMENTS SUR L'HISTOIRE DE LORRAINE, 27). 


\section{Les communautés religieuses}

Pour étudier la répartition régionale des monastères et collégiales ainsi que le processus de leur fondation, nous avons dressé un catalogue des communautés religieuses dans l'espace lotharingien ${ }^{30}$. Parmi les 148 établissements répertoriés figurent quatre maisons appartenant aux diocèses de Reims et de Langres ${ }^{31}$. Ces données permettent de s'interroger sur des phénomènes de dynamique spatiale mais aussi temporelle. Le tableau intitulé «Monastères et collégiales en Lotharingie» résume les résultats de ce travail.

Monastères et collégiales en Lotharingie

\begin{tabular}{|c|c|c|c|c|c|c|}
\hline $\begin{array}{l}\text { Province } \\
\text { ecclésias- } \\
\text { tique }\end{array}$ & Diocèse & $\begin{array}{l}\text { Nombre } \\
\text { d'établis- } \\
\text { sements }\end{array}$ & urbains & ruraux & $\begin{array}{l}\text { Fonda- } \\
\text { tion avant } \\
855\end{array}$ & $\begin{array}{l}\text { Fonda- } \\
\text { tion après } \\
855\end{array}$ \\
\hline & Utrecht & 5 & 2 & 3 & 2 & 3 \\
\hline & Liège & 26 & 4 & 22 & 18 & 8 \\
\hline & Cologne & 13 & 11 & 2 & 7 & 6 \\
\hline $\begin{array}{l}\text { Total } \\
\text { Cologne }\end{array}$ & & 44 & 17 & 27 & 27 & 17 \\
\hline \multirow{2}{*}{ (Reims) } & Cambrai & 25 & 5 & 20 & 18 & 7 \\
\hline & (Reims) & 2 & & 2 & 1 & 1 \\
\hline \multirow[t]{5}{*}{ Total Nord } & & 71 & 22 & 49 & 46 & 25 \\
\hline & Trèves & 17 & 7 & 10 & 13 & 4 \\
\hline & Metz & 15 & 6 & 9 & 10 & 5 \\
\hline & Verdun & 4 & 2 & 2 & 3 & 1 \\
\hline & Toul & 16 & 3 & 13 & 11 & 5 \\
\hline $\begin{array}{l}\text { Total } \\
\text { Trèves }\end{array}$ & & 52 & 18 & 34 & 37 & 15 \\
\hline (Mayence) & Strasbourg & 15 & 3 & 12 & 12 & 3 \\
\hline (Besançon) & Bâle & 8 & 1 & 7 & 8 & \\
\hline (Lyon) & (Langres) & 2 & & 2 & & 2 \\
\hline Total Sud & & 77 & 22 & 55 & 57 & 20 \\
\hline $\begin{array}{l}\text { Total } \\
\text { Lotharingie }\end{array}$ & & 148 & 44 & 104 & 103 & 45 \\
\hline \multirow{3}{*}{$\begin{array}{l}\text { Pourcen- } \\
\text { tage } \\
\quad \text { Nord } \\
\text { Sud }\end{array}$} & & 100 & 30 & 70 & 70 & 30 \\
\hline & & 48 & 31 & 69 & 65 & 35 \\
\hline & & 52 & 29 & 71 & 74 & 26 \\
\hline
\end{tabular}

30 Schneider, Suche, annexe 6, p. 533-541. Ce catalogue doit beaucoup aux travaux d'A.

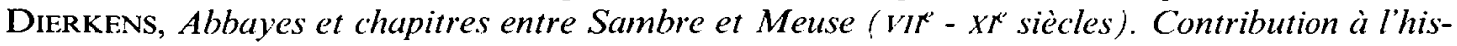
toire religieuse des campagnes du Haut Moyen Age, Sigmaringen, 1985 (BEIHEFTE DER Francia, 14) et d'A.-M. HelvéTIus, Abbayes, évêques et lä̈ques. Une politique du pouvoir en Hainaut au moyen age ( $v I T^{\circ}-X t^{\prime}$ siècle), Bruxelles, 1994 (Crédit Communal. Coll. Histoire in-8ª 92).

31 Montfaucon, Mouzon; Poulangy, Varennes. Au sujet de l'identification problématique de Poulangy voir Schneider, Suche, p. 75-76, 104. 
Pour la séparation entre Sud et Nord nous avons emprunté la frontière bien connue depuis l'époque romaine entre les provinces de Germanie première et seconde. Elle est marquée par un affluent du Rhin (le Vinxtbach) à l'est et devint celle entre les provinces ecclésiastiques de Trèves et de Cologne et, plus tard, plus ou moins celle des duchés de Haute et de Basse-Lotharingie.

La répartition des maisons est assez équilibrée. S'agissant du nombre de chartes, on constate une légère prépondérance du Sud (77 contre 71). On n'est pas surpris de cette relation qu'on aurait peut-être même attendu plus prononcée. Plusieurs facteurs jouent ici. Tout d'abord, les fameux monastères vosgiens (Annegray, Luxeuil, Lure, Faverney) ne font pas partie de l'espace lotharingien tel que nous l'avons défini. Ensuite, il faut souligner que le Sud a été romanisé et christianisé plus précocement et plus intensément que le Nord. En revanche, il ne faut pas oublier qu'une grande partie de l'espace néerlandais-belgo-rhénan a été la zone d'action de la famille carolingienne bien avant l'accès à la royauté ${ }^{32}$. Ceci est mis en évidence par le nombre de communautés situées dans les diocèses de Cambrai et de Liège ( 25 et 26 ).

La répartition entre les communautés rurales et les fondations urbaines, si fait urbain il y avait, est à peu près la même au Nord et au Sud (respectivement 70 et $30 \%$ ). C'est le cas de Cologne qui fait exception. Plus de $80 \%$ des communautés du diocèse de Cologne se situent dans la cité même, une proportion inverse de celle que l'on constate pour les diocèses de Cambrai, de Liège et aussi d'Utrecht. Comment s'explique cette inversion? Elle est avant tout due à un contraste structurel. Les anciennes villes romaines le long du Rhin - Cologne, Bonn, Xanten - ont été plus fréquentées et furent, par conséquent, plus développées que Liège et Cambrai. La tradition urbaine qui commence seulement à se développer ailleurs est assez nette ici. Il faut rappeler que Cambrai est devenue siège d'évêché vers 600 et qu'elle a pris la place de l'ancienne cité romaine qu'était Bavay ${ }^{33}$. Pour Liège, nous savons grâce aux travaux de Jean-Louis Kupper que c'est à partir de 800 environ que l'évêque de Tongres y choisit sa résidence principale $^{34}$. Les données pour Utrecht, évêché destiné à la mission du

32 M. WERNER, Der Lütticher Raum in frühkarolingischer Zeit. Untersuchungen zur Geschichte einer karolingischen Stammlandschaft, Göttingen, 1980 (VERÖFFENTLICHUNGEN des Max-Planck-Instituts für Geschichte, 62) - A. Dierkens, La christianisation des campagnes de l'Empire de Louis le Pieux. L'Exemple du diocèse de Liège sous l'épiscopat de Walcaud (c. 809 - c. 831 ), dans P. Godman, R. Collins dir., Charlemagne's Heir. New Perspectives on the Reign of Louis the Pious (814-840), Oxford, 1990, p. 309-329.

33 Topographie chrétienne des cités de la Gaule des origines au milieu du vIIr siècle, vol. 14: Province ecclésiastique de Reims (Belgica secunda), Paris, 2006, p. 101-102. - Au sujet de saint Géry qui déménagea le siège épiscopal d' $\Lambda$ rras à Cambrai voir $R$. SCHIEFFER, Die Entstehung von Domkapiteln in Deutschland, Bonn, 1982 (BONNER HistorisCHE ForsCHUNGEN, 43), p. 137-139.

34 J.-L. KUPPER, Liège au viri ${ }^{\mathrm{e}}$ siècle. Naissance d'une ville sanctuaire, dans M. Polfer dir., L'évangélisation des régions entre Meuse et Moselle et la fondation de l'abbave d'Echternach ( $V^{\prime}-I X^{\prime}$ siècle), Luxembourg, 2000 (Publications DU CLUDEM, 16), p. 355-364; 
temps de Willibrord, ne permettent pas d'évaluation ${ }^{35}$. Comme à Bâle ${ }^{36}$, le siège épiscopal s'est établi au cours du vili ${ }^{\mathrm{e}}$ siècle sans connaître de véritable évolution urbaine avant longtemps. Force est donc de constater une sorte de dynamique urbaine dans les anciennes cités romaines près du Rhin par rapport à une tradition monastique rurale dans le reste des anciens Pays-Bas; une dynamique qui deviendra plus marquée vers la fin

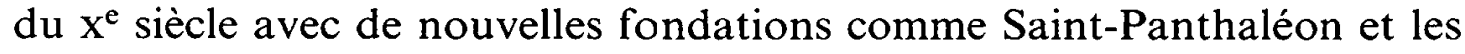
Saints-Apôtres à Cologne, ou Saint-Laurent à Liège ${ }^{37}$.

Passons à une interrogation diachronique du tableau. Au premier coup d'œil on constate 25 nouvelles fondations au Nord par rapport à 20 pour le Sud. La relation entre les anciennes fondations, nées avant le début de notre période, et les nouvelles maisons montre que le paysage des institutions religieuses en Belgique première et en Alsace est plus traditionnel et plus homogène, mais aussi, on le verra, moins dynamique. On observe ici un phénomène semblable en ce qui concerne la localisation, rurale ou urbaine, des établissements. Sur les 25 nouvelles institutions de la partie nord on distingue huit communautés fondées en ville: il s'agit, à Cologne, de Saint-André, Sainte-Cécile, Sainte-Marie-en-Capitole, Saint-Séverin, Sainte-Ursule et Saint-Martin (Groß-St. Martin); à Liège, de Saint-Pierre et, à Utrecht, de Sainte-Marie. Plus d'un tiers des communautés de Basse-Lotharingie (35\%) sont des fondations nouvelles alors que celles-ci ne représentent qu'un quart des établissements au Sud $(26 \%)$. Le diocèse le plus dynamique est Cologne avec six nouvelles contre sept anciennes maisons et, dans une moindre mesure, Utrecht avec trois contre deux. Notons pour l'instant cette position à part de ces deux villes.

Pour résumer l'analyse du premier tableau, on constate une dynamique qu'on pourrait dire de rattrapage assez marquée dans l'espace de la future Basse-Lotharingie. Parmi les 103 monastères fondés avant 855 en Lotharingie, $44 \%$ se trouvent au Nord. Sur les 45 fondations nées à partir de cette date, la part septentrionale s'élève à $56 \%$. $38 \%$ de ces nouvelles communautés sont situées dans la province ecclésiastique de Cologne, plus précisément huit dans le diocèse de Liège, six dans le diocèse de Cologne et trois dans celui d'Utrecht. Cette dynamique semble principalement due aux grandes familles nobles qui continuent à établir des communautés religieuses sur leurs terres. Le rôle des évêques et des rois

IDEM, Aux origines de la cité de Liège. Sur deux chartes inédites de 1171 et 1266, BuLLETIN DE la Commission royale D'histoire, 175, 2009, p. 321-342 - Cf. F. Dhein, Lüttich. Inszenierung einer Bischofsstadt an der Wende zum 11. Jahrhundert, dans J. JARNUT, A. KöB, M. WEMHOFF dir., Bischöfliches Bauen im 11. Jahrhundert, Munich, 2009 (MitrelalterSTUdiEN, 18), p. 191-200.

35 VAN VLIET, In kringen, p. 203-204 - Cf. bientôt W. VAN EgmOND, Utrecht in the Early' Middle Ages: Historical Research and Problems (sous presse).

36 Helvetia Sacra, N. F., A. Bruckner dir., vol. 1.1, Berne, 1972, p. 128.

37 O. ENGELS, Klöster und Stifte von der Merowingerzeit bis um 1200, Bonn, 2006 (Geschichtlicher Atlas der Rheinlande, 9.2), p. 46-50 - Dhein, Lüttich, p. 195. 
est nettement moins important. A Utrecht, c'est l'évêque Baldéric qui fonde Sainte-Marie (Oudmunster) dans sa cité, mais les monastères de Tiel et d'Egmond ont été fondés par la famille comtale. On voit ici, avec le comes Dirk, les débuts du comté de Hollande ${ }^{38}$. Si l'évêque Richer fonde Saint-Pierre de Liège et Sainte-Marie à Huy sur la Meuse, ce sont des grandes familles nobles qui sont à l'origine de six autres communautés: Brogne, Dinant, Gembloux, Salles, Hastière et Waulsort ${ }^{39}$.

Revenons au cas de Cologne. La remarquable dynamique urbaine qu'on a pu y constater me semble liée à la présence ottonienne, plus particulièrement à l'action de l'archevêque Brunon (953-965), frère de l'empereur. Toutes les nouvelles fondations dans ce diocèse sont les siennes. En même temps, nous tenons là un deuxième élément d'explication pour la dynamique particulière à Cologne mais aussi à Utrecht: l'influence des rois ottoniens qui est évidente à Cologne et qui se manifeste à Utrecht par les nombreux diplômes en faveur de l'évêché frison. On en compte treize pour la période étudiée ici. Parmi tous les destinataires de diplômes en Lotharingie, seul le monastère de Prüm en reçoit plus ${ }^{40}$. Brunon apparaît comme le précurseur d'un mouvement qui se manifestera à Liège par le bouquet de collégiales dont l'évêque Notger dote sa cité ${ }^{4 !}$.

\section{Une analyse quantitative}

On a dit que l'époque carolingienne a été le «moment fondateur dans l'histoire de l'acte royal/impérial ${ }^{42}$. Du règne d'Arnulf, dernier empereur carolingien (à l'exception de Louis, petit-fils de Louis d'Italie, qui fut aveuglé en 905), sont conservés 237 diplômes; pour la même période on en compte 80 dans le futur royaume de France ${ }^{43}$. Pour les cinq années du règne de Zwentibold en Lotharingie, on dispose de 28 diplômes ${ }^{44}$. Compte tenu de l'espace occupé par ces trois royaumes, fluctuant de surcroît avec la création du regnum lotharingien en 895 et la promotion d'Arnulf au titre impérial en 896, il est difficile de comparer ces chiffres.

38 Recueil Charles III, éd. LAUER, n 121 ; MGH DD O.I, nº 124 - VAN VlifT, In kringen, p. $160-161$.

39 Brogne: J.-P. Devroey, Les premiers domaines de l'abbaye Saint-Pierre de Brogne ( $\mathrm{X}^{\circ}$ - $\mathrm{XII}^{*}$ siècles), dans La seigneurie rurale en Lotharingie. Luxembourg, 1986, p. 51-67 Dinant: J. Sfmmler, Vita Religiosa an Mosel und Saar ca. 650 - ca. 850, dans Polfer, L'évangélisution, p. 15-36 - Salles: Helvétıus, Abbayes - Gembloux, Hastière et Waulsort: Dierkens, Abbayes.

40 MGH DD Lo.II, n 7, DD LDt., $\mathrm{n}^{\circ} 68$, DD Zw, n 9, DD Ko.I, $\mathrm{n}^{\circ}$ 24, DD H.I, $\mathrm{n}^{\circ} 27$, DD O.I, n6, 19, 58, 62, 98, 112, 124, 164 - SchNeIder, Suche, annexe 5, p. 530-532.

41 J.-L. Kupper, Notger de Liège. Un évêque lotharingien aux alentours de l'an mille, dans H.-W. Herrmann, R. SchneIder dir., Lotharingia. Une région au centre de l'Europe autour de l'an mil, Sarrebruck, 1995, p. 143-154 - DHEIN, Lüttich.

42 Guyotjeannin, Pycke, Tock, Diplomatique, p. 105.

4.3 Guyotjeannin, Pycke, Tock, Diplomatique, p. 106.

44 MGH DD Zw, no 1-28. 
Toujours est-il que le nombre de 28 diplômes sur cinq ans rejoint plutôt la situation du royaume d'Eudes et de Charles le Simple avant 900 que celle beaucoup plus riche de la Francie orientale. On verra comment la situation se présente à travers les chartes.

\section{Chartes en Lotharingie}

\begin{tabular}{|r|c|c|c|c|c|c|}
\hline & \multicolumn{3}{|c|}{ Corpus restreint } & \multicolumn{3}{c|}{ Corpus entier } \\
\cline { 2 - 8 } & $855-899$ & $900-925$ & $926-959$ & $855-899$ & $900-925$ & $926-959$ \\
\hline Total & 67 & 29 & 74 & 89 & 43 & 132 \\
\hline \multirow{2}{*}{ Nord } & 20 & 12 & 29 & 28 & 16 & 45 \\
Sud & 47 & 17 & 45 & 61 & 27 & 87 \\
\hline Relation Nord-Sud & $1: 2$ & $2: 3$ & $2: 3$ & $1: 2$ & $2: 3$ & $1: 2$ \\
\hline
\end{tabular}

Ce deuxième tableau présente une analyse quantitative des chartes répertoriées. Il comprend 264 chartes dont l'émetteur où le destinataire se trouve en Lotharingie ${ }^{45}$. L'attribution d'une charte au Nord ou au Sud, selon la frontière provinciale déjà évoquée plus haut, tient compte en premier lieu de l'émetteur, puis du destinataire et, si les deux ne sont pas identifiables, en troisième lieu de la tradition manuscrite (cartulaire etc.). Les chartes sont réparties en trois tranches chronologiques. On distingue du corpus entier, à droite, un corpus restreint en grisé qui comprend seulement les chartes privées au sens propre, c'est-à-dire sans les actes des grands nobles, tels les évêques, les comtes et la reine Richilde ${ }^{46}$. Cette distinction n'est pas sans poser des problèmes de méthode, mais l'analyse du tableau montrera que bien avant l'existence de véritables chancelleries épiscopales ou seigneuriales, la production de chartes se fait de plus en plus auprès des grands du royaume.

Sur ces 264 chartes, il y a 79 actes d'évêques conservés ${ }^{47}$. La moitié de ces chartes épiscopales ont été émises par les évêques de Trèves et de Metz (20 chacun). De leurs collègues de Cologne et de Toul on a conservé 14 et 13 chartes. Pratiquement la seule charte connue de l'évêque de Liège pour notre période date de 932 : le 16 novembre de cette année-là,

45 Sur les sources et les principes de la composition du tableau voir SCHNEIDER, Suche, annexe 4, p. 500-519. Je remercie Benoît-Michel Tock, Université de Strasbourg, et JeanBaptiste Renault, Université de Nancy II, de m'avoir donné la possibilité d'utiliser la Base des actes originaux conservés en France antérieurs à 1121 (Base ARTEM) avant qu'elle ne soit mise en ligne: http://www.cn-telma.fr/artem - Cf. La diplomatique française du Haut Moyen Age. Inventaire des chartes originales antérieures à 1121 conservées en France, B.-M. Tock dir., 2 vol., Turnhout, 2001.

46 Pour la discussion sur l'acte privé et le choix des trois créneaux voir Tock, L'acte prive, p. 500-502 et tableau p. 505 -- Cf. DESPY, Les chartes privées.

47 SCHNeIder, Suche, annexe 4.1, p. 520-522. 
l'évêque Richer consacre un autel à la Trinité dans sa cathédrale ${ }^{48}$. On peut constater que les quatre scriptoria épiscopaux de la province ecclésiastique de Trèves font preuve d'une activité continue, bien avant le Nord lotharingien. Il y a très peu de documentation pour les grands laïcs, la plupart des actes conservés provenant d'un scriptorium monastique comme le montre les chartes du duc Gislebert données par lui en qualité d'abbé 49 .

Si l'activité des grands laïcs en matière diplomatique dans notre période apparaît assez faible, le corpus étudié témoigne toutefois de l'activité de femmes dans la production de chartes. Sur la totalité du corpus, 33 chartes mettent en évidence des femmes en tant qu'émettrice (19) ou destinataire principale $(14)^{50}$. Plus précisément, nous avons neuf chartes pour le Nord lotharingien et 24 pour le Sud. Cette répartition correspond tout à fait aux proportions résumées dans le deuxième tableau. La relation entre les chartes émises et chartes reçues montre cependant que les femmes semblent avoir pris une part plus importante en tant qu'émettrices dans la partie septentrionale avec sept chartes sur neuf au Nord par rapport au Sud lotharingien avec seulement la moitié des 24 chartes.

A côté des femmes plus ou moins inconnues des actes de précaire et de donation, le corpus renferme cinq chartes relatives à trois femmes nobles. Celles-ci sont Richilde, la veuve de Charles le Chauve ${ }^{51}$, la comtesse Hildegonde qui fonde le prieuré d'Amel en dépendance de Gorze ${ }^{52}$, et la comtesse Ève. De cette Ève, mère du futur archevêque de Reims Odelric, nous disposons de quatre chartes, dont un faux. Eve apparaît pour la première fois en 942 dans un acte d'échange avec l'abbé de Saint-Evre de Toul ${ }^{53}$. Notons en passant la mention du titre de comtesse qui n'est pas encore courant dans les chartes. ${ }^{54}$ Le recueil de textes narratifs et

48 Limburgsche oorkonden, éd. J. CoEnen, vol. 1, Maaseik, 1932, $\mathrm{n}^{\circ} 111$. L'acte est transmis par Anselme de Liège, Gesta episcoporum Leodiensium, éd. R. KOEPKE, dans MGH SS 7, Hanovre, 1846, p. 200-201. Voir en dernier lieu Fl. Close, L'octave de la Trinité du calendrier liégeois, LE MOYEN AGE, 110, 2004, p. 628. Je tiens à remercier Alexis Wilkin, Université Libre de Bruxelles, qui m'a communiqué ces informations. - Après 959: Recueil Stavelot, éd. Halkin, Roland, no 79 a. 960 (Everacre).

49 SCHNeIder, Suche, annexe 4.2. Pour Gislebert voir Recueil Stavelot, éd. Halkin, ROLAND, no 53-58, 63; Urkundenbuch zur Geschichte der mittelrheinischen Territorien, éd. H. Beyer, L. Eltester, A. Goerz, Aalen, 1974, vol. I, no 165, 166, 169.

50 Schneider, Suche, annexe $4, \mathrm{n}^{\circ} 3,18,32,49,58,70,74,81,87,97,105-106,118,133$, 149, 166, 170-174, 187, 195-196, 202, 205, 210, 213, 219, 227, 232, 244, 263.

51 Cartulaire de l'abbaye de Gorze. Ms. 826 de la Bibliothèque de Metz, éd. A. D'HerBOMEz, Paris, 1898 (MetTensia, 2), nº 87 et 88 a. 910.

52 Cartulaire Gorze, éd. D’Herbomez, n 107 a. 959.

53 Chartes de l'abbaye Saint-Evre de Toul des origines à 1228, éd. M. SCHAEFFEr, Thèse de $3^{e}$ cyclc, Université de Nancy II, 1983, $\mathrm{n}^{\circ} 17$ :... inter praefatu[m] Archembaldum abbatem religio[si]ssimum atque Evam cometissam ... (p. 127).

54 Pour l'année 859, L. Morelle a signalé une charte dont il a pu confirmer l'authenticité qui a été disputée: ego Vuarimburgis, gratia Dei comitissa (Paris, AN, K $10 \mathbf{n}^{\circ} 6^{2}$ ). L. Morelle, Histoire des archives ecclésiastiques médiévales, École pratique des hautes ÉTUdES, SECTION DES SCIENCES HISTORIQUES ET PHILOLOGIQUES, LIVRET-ANNUAIRE, 17 (2001- 
diplomatiques qu'on appelle traditionnellement le Petit Cartulaire de Saint-Arnoul contient trois chartes qui mentionnent la comtesse Ėve en tant que donatrice de la villa de Lay-St-Christophe à l'abbaye de SaintArnoul: deux chartes au nom d'Ève et son fils Odelric, dont la première doit être considérée comme fausse à cause de la mention d'Odelric en tant qu'archevêque de Reims, office qu'il n'exerçait pas avant $962^{55}$; puis une troisième charte donnée neuf ans plus tard par Udelricus, filius Eve comitisse $^{56}$.

Le corpus restreint, marqué en grisé, présente 170 chartes réparties en trois tranches chronologiques ${ }^{57}$. Parmi les émetteurs figurent 33 abbés, un rector, quatre prieurs (Gorze, Stavelot, Echternach) et deux primicerii de Toul : cela fait 40 . Restent 130 chartes, soit la moitié du corpus entier. Ce sont des personnes dont on ne sait pas grand-chose. Il s'agit là pour la plupart de donations et de précaires pour des églises et surtout des monastères et collégiales.

La production diplomatique dans le Sud, la future Haute-Lotharingie, fait à peu près le double de celle du Nord. Ce constat vaut pour le corpus entier et, avec quelques nuances, pour le corpus restreint en grisé. Dans la deuxième tranche chronologique on observe une baisse statistique assez nette. Cette chute du chiffre des actes conservés au début $\mathrm{du} \mathrm{x}^{\mathrm{e}}$ siècle, suivie d'une véritable explosion du matériel diplomatique est en accord avec nos connaissances sur la production écrite en général. L'évaluation statistique des actes d'échange présentée lors d'un colloque récent mène à un constat comparable, sauf en ce qui concerne Saint-Gall et la Bavière (hormis Freising) ${ }^{58}$. Après cette baisse, on observe un phénomène de redémarrage qui est plus marqué dans le corpus entier que dans le corpus restreint: du début à la fin de la période, on passe de 89 actes à 132 pour le corpus entier et de 67 à 74 pour le corpus restreint. Si on se focalise sur la différence entre Nord et Sud, on constate que la baisse statistique est plus forte dans le Sud où elle tombe au-dessous de la moitié, plus prononcée

2002), 2003, p. 201-204 - En 923, on trouve l'intitulation de la veuve de Richard, duc de Bourgogne: ego, Adeleydis, superna disponente pietate, condam comitissa (ARTEM n ${ }^{\circ} 609$ ). Cf. R. LE JAN, Famille et pouvoir dans le monde franc (VIT - Xe siècle). Essai d'anthropologie sociale, Paris, 2003 (HISTOIRE ANCIENNE ET MÉdí́vale, 33), p. 358-360.

55 Le souvenir des Carolingiens à Metz au Moyen Age. Le Petit Cartulaire de SaintArnoul, éd. M. Gaillard, Paris, 2006 (Textes et doCUments d'histoire médiévale, 6 ), n 60 et 61 : Metz 950, 15 et 16 août; n०61: Eva, nobilissimi condam comitis Hugonis uxor, et filius meus dilectissimus Udelricus, iam favente Dei clementia in ordine clericatus constitutus. Sur la fausseté du n $\mathrm{n}^{\circ} 60$ ibidem, p. 211 (M. Parisse) - M. PARISSE, La Lorraine du IX $^{\mathrm{e}}$ au XI $\mathrm{XI}^{\mathrm{e}}$ siècle. Les travaux de M. Hlawitschka, ANNAles DE L'EST, 23, 1971, p. 106-107 - A. CALMET, Histoire de Lorraine, Nancy, 1745-1757, réimpr. Paris, 1973, vol. 2, col. CXCVI-CXCVIII, notes $s$ et $t-\mathrm{CF}$. ARTEM $\mathrm{n}^{\circ} 308$ et Urkunden- und Quellenbuch, éd. WAMPACH, vol. $1, \mathrm{n}^{\circ} 162$.

56 Petit Cartulaire, éd. Gaillard, nº 62.

57 SCHNEIDER, Suche, annexe 4.3.

58 «L'acte d'échange, du viII ${ }^{\mathrm{e}}$ au XII ${ }^{\mathrm{e}}$ siècle», Limoges, 11-13 mars 2010. Publication des actes à paraître sous la direction de Ph. Depreux et I. FEEs - Cf. GuYotjeannin, Penuria, et Bougard, Mise en écriture. 
encore dans le corpus restreint (de 47 à 17). La proportion initiale de 1 à 2 sera atteinte de nouveau après la période médiane, mais pour le corpus entier, et pas pour le corpus restreint, en grisé, où elle sera alors de 2 à 3. L'effort de redémarrage y paraît alors plus important au Nord où le nombre d'actes est supérieur par rapport à la situation d'avant la baisse (20 - 29) tandis qu'au Sud il n'atteint pas celui d'avant $(47-45)$.

Les données résumées dans le deuxième tableau permettent de parler d'une évolution modeste mais continue pour l'espace des anciens PaysBas et de la Rhénanie. L'explosion de la production diplomatique après 925 s'explique par différents phénomènes. Dans le Sud lotharingien, ce sont surtout les évêques qui ont pu mener une politique personnelle qui se manifeste dans leurs actes compris dans le corpus entier. L'analyse des actes épiscopaux montre que ce constat s'applique surtout à la Lorraine mais aussi au diocèse de Cologne en Basse-Lotharingie. En revanche, dans les vastes régions situées au Nord et à l'Ouest de la Meuse comme la Toxandrie, les évêques ont dû laisser le champ libre aux grands laïcs. A travers les chartes se manifeste la même dynamique de rattrapage déjà observée à l'exemple des fondations d'institutions de vita communis. Pour les familles puissantes, ces régions là semblent avoir représenté en quelque sorte un nouveau monde moins contrôlé.

On insistera un instant sur la famille des Régnier ${ }^{59}$. Régnier $\mathrm{I}^{\mathrm{er}}$, 'au long cou', et plus encore son fils Gislebert sont parmi les personnages laïcs les plus puissants dans la première moitié $\mathrm{du} \mathrm{x}^{\mathrm{e}}$ siècle pour notre espace lotharingien. Au premier regard on ne retrouve pas cette importance dans le corpus. Alors n'étaient-ils pas aussi éminents que l'on a toujours pensé? $\mathrm{Si}$, mais dans la documentation diplomatique ils n'apparaissent pas en premier lieu en tant que comte ou duc mais dans leur fonction abbatiale, leurs actes étant rédigés dans un de leurs monastères. On trouve 14 actes émis par Régnier $\mathrm{I}^{\mathrm{er}}$ ou par son fils Gislebert et huit autres qui les mentionnent en tant que témoin ${ }^{60}$. Notons que 13 de ces 22 actes sont conservés dans le cartulaire de Stavelot qui est une source des plus riches pour notre question. ${ }^{61}$

59 A.-J. BiJsterveld, Les sépultures des comtes de la Meuse inférieure: le cas des Régnier et des Baldéric ( $\mathrm{x}^{\mathrm{e}}$ siècle), des comtes de $\mathrm{Looz}\left(\mathrm{xI}^{\mathrm{e}}\right.$ siècle) et des comtes de Gueldre (XII ${ }^{\mathrm{e}}$ - XIV $\mathrm{V}^{\mathrm{e}}$ siècles), dans M. MARGUE dir., Sépulture, mort et représentation du pouvoir au moyen âge, Luxembourg, 2006 (Publications du CLUDEM, 18), p. 375-404 - G. DeSPY, Abbatiat laïc et manipulations foncières en Lotharingie vers 900 : la "charte de précaire" du duc Gislebert de 928, dans La Belgique rurale du Moyen Age à nos jours, Mél. J.-J. HoEBANX, Bruxelles, 1985 (Faculté de Philosophie et Lettres, 95), p. 19-28 - A. Dierkens, M. Margue, Memoria ou damnatio memoriae? L'image de Gislebert, duc de Lotharingie († 939), dans Retour aux Sources, Mél. M. PARISSE, Paris, 2004, p. 869-890 - R. LE JAN, L'Aristocratie Lotharingienne: Structure interne et conscience politique, dans HERRMANN, Lotharingia, p. 71-88.

60 SCHneider, Suche, annexe 4, no 33, 96, 99, 101, 107 (Régnier); no 113-114, 122, 134-136, 139, 142, 147, 156 (Gislebert); en tant que témoins : nº 100 (Régnier), 144, 152-153, 155, 157-158, 173 (Gislebert).

61 Recueil Stavelot, éd. Halkin, Roland, n 49-51, 53-61, 63. 


\section{Conclusions}

L'exemple des Régnier donne lieu à une première conclusion concernant le sujet du colloque, les chancelleries princières. Voici une famille qui comptait assurément parmi les premières durant la période étudiée. Apparentés aux rois carolingiens et ottoniens, ils ont réussi à garder les abbatiats de Stavelot, de Saint-Servais de Maastricht, d'Echternach et de Saint-Maximin de Trèves sur plusieurs générations. Une bonne vingtaine d'actes témoigne de leur influence politique; si on regarde leur production diplomatique, les Régnier égalent un évêque lorrain et ils paraissent bien plus importants que l'évêque de Liège, de Cambrai ou celui d'Utrecht. Toutefois, ils n'ont pas établi une structure de production écrite qui leur fût propre. L'exemple du duc de Normandie qui, au $\mathrm{xI}^{\mathrm{e}}$ siècle, n'aurait pas disposé de chancellerie ni de sceau avant de devenir roi d'Angleterre confirme que la situation ne va pas changer de si tôt ${ }^{62}$.

Comme bien d'autres grands, Régnier et Gislebert, quand ils veulent mettre en écrit quelque action juridique, se tournent vers un scriptorium monastique. Il y a néanmoins quatre chartes qui ne semblent pas provenir d'un monastère. Il s'agit de trois actes conservés dans le cartulaire de Stavelot, à savoir les $n^{\text {os }} 51$ (Huy, a. 911), 55 (Namur, a. 922), et 56 (Wellin, a. 926). Il faut leur ajouter une charte de précaire de l'an 928 qui a souvent été considérée comme fausse mais qui, d'après Georges Despy, aurait été dressée pour acter un compromis négocié par le roi Henri $\mathrm{I}^{\mathrm{er}}$ entre Gislebert et l'archevêque Rutger de Trèves. Gislebert y est mentionné en tant que rector de Saint-Servais de Maastricht ${ }^{63}$. La question posée par Despy en 1960 dans le Bulletin de la Commission royale d'Histoire semble toujours valable ${ }^{64}$ : comment faut-il imaginer les conditions concrètes de l'émission d'une charte dans le castrum de Huy, de Namur ou dans la villa Wellin dans les Ardennes?

La présente étude a montré qu'il convient d'insister davantage sur les lieux d'émission des actes. Une deuxième carte a été dressée afin de permettre une analyse croisée du témoignage des actes et de la répartition des établissements religieux. Sur une échelle à trois niveaux on distingue quatre lieux d'émission importants avec 16 à 20 chartes émises, puis Trèves et Toul avec 13 et 14 , et enfin 18 maisons avec peu de documentation conservée. La répartition des lieux de production diplomatique y apparaît relativement équilibrée. Parmi les quatre grands centres deux se

\footnotetext{
62 Recueil des actes des ducs de Normandie de 911 à 1066, éd. M. Fauroux. Caen, 1961 (Mémoires de la Société des Antiquaires de Normandie, 36), p. 41-43, $45-47$ Guyotjeannin, Pycke, TOCK, Diplomatique, p. 111.

63 Urkundenbuch, éd. Beyer, Eltester, GOERz, no 169: Gysalbertus gratia dei dux rectorque sancte Traiectensis ecclesie. Cf. DESPY, Abbatiat - Le même titre est conservé pour son père Régnier: WAMPACH, Echternach, n $149,150,155,163$.

${ }_{64}$ G. Despy, A. Joris, Etude sur une charte privée, rédigée à Huy en 866 , pour l'abbaye de Kornelimünster, B.C.R.H., 126, 1960, p. 103-134.
} 
trouvent au Nord (Cologne et Stavelot). Dans l'espace des anciens PaysBas on trouve davantage de petits scriptoria, dont on a conservé juste un ou deux actes pour notre période.

Pour des raisons de méthode, aucune distinction n'a été faite entre l'institution de l'évêché et un ou plusieurs monastères ou collégiales dans la cité épiscopale. A côté des institutions mentionnées en tant que lieu d'émission à la fin d'une charte il y a une centaine d'actes dans le corpus sans lieu indiqué. Il n'est pas nécessaire d'insister ici sur le fait que ce manque de renseignements est partiellement imputable à l'état de la tradition. Pour les 20 chartes conservées dans le Liber aureus Epternacensis, tout ce qui nous reste de l'eschatocole est une brève ligne de datation; même pour les actes qui mentionnent les abbés d'Echternach il n'y a pas plus de renseignements indiqués ${ }^{65}$. En dépit du fait qu'aucun des actes édités par Camillus Wampach ne donne la mention actum Epternacense, cette abbaye est bien représentée sur la carte parce qu'il paraît légitime de supposer qu'un certain nombre d'actes ont été rédigés dans le scriptorium d'Echternach. L'abbaye de Bouxières présente un cas similaire ${ }^{66}$. Sur la carte figure également le monastère de Mettlach, même s'il n'est pas certain que la charte qui nous renseigne sur la consécration de l'église à Udera par l'archevêque de Trèves et en présence du duc Gislebert, fut écrite à Mettlach ${ }^{67}$. En tout cas il semble que l'église en question ait appartenu à Mettlach et que la charte ait été copiée dans le cartulaire de Mettlach.

Restent en outre 27 chartes dont l'Actum ne mentionne pas un monastère mais une église, un castrum ou une villa. Parmi les 23 différents lieux, 15 se trouvent au Sud et huit au Nord lotharingien: Aix-la-Chapelle, Huy, Namur, Waha, Wellin ${ }^{68}$ et, en Frise, Gannita, Hlara et Hlegilo ${ }^{69}$. Cela n'empêche pas que ces chartes soient sorties d'un scriptorium monastique mais la réflexion sur ces lieux d'émission reste une piste à explorer ${ }^{70}$.

65 WampaCH, Echternach, n 150,163 (Régnier), no 152 (Carloman), nº 154 (Adélard), $\mathrm{n}^{\circ} 161$ (Wigéric).

66 Les origines de l'abbaye de Bouxières, éd. Bautrer, à lire avec K. Oschema, Zur Gründung des Benediktinerinnenklosters Notre-Dame de Bouxières. Eine wiedergefundene Urkunde des 10. Jahrhunderts, MitTEILUNGEN DES INSTITUTS Für ÖSTERREICHISCHE GeSCHICHTSFORSChUNG, 110, 2002, p. 182-190.

67 A. GoERz, Regesten der Erzbischöfe zu Trier von Hetti bis Johann II. 814-1503, Aalen, 1984 (première édition 1859-1861), a. 932, p. 3.

68 Recueil Stavelot, éd. Halkin, Roland, no 45, 46, 51 (Huy), n 55 (Namur), n ${ }^{\circ} 56$ (Wellin), n० 61 (Waha), n 74 (Aix); Chartes de l'abbaye de Waulsort. Etude diplomatique et Edition critique, vol. 1 (946-1199), éd. G. DESPY, Bruxelles, 1957, no 2 (Namur)-Pour l'acte d'Aix-la-Chapelle voir supra note 28.

69 Urkundenbuch für die Geschichte des Niederrheins, éd. Th. J. LACOMBLET, vol. 1, Düsseldorf, 1840, $\mathrm{n}^{\circ} 65$ (Hlara et Hlegilo); Oorkondenboek der graafschappen Gelre en Zutfen tot op den slag van Woeringen, 5 Juni 1288, éd. L. A. J. W. Sloet, vol. 1, La Haye, 1872-1876, n' 52, 65 (Gannita).

70 Cf. MORElle, Incertitudes. 


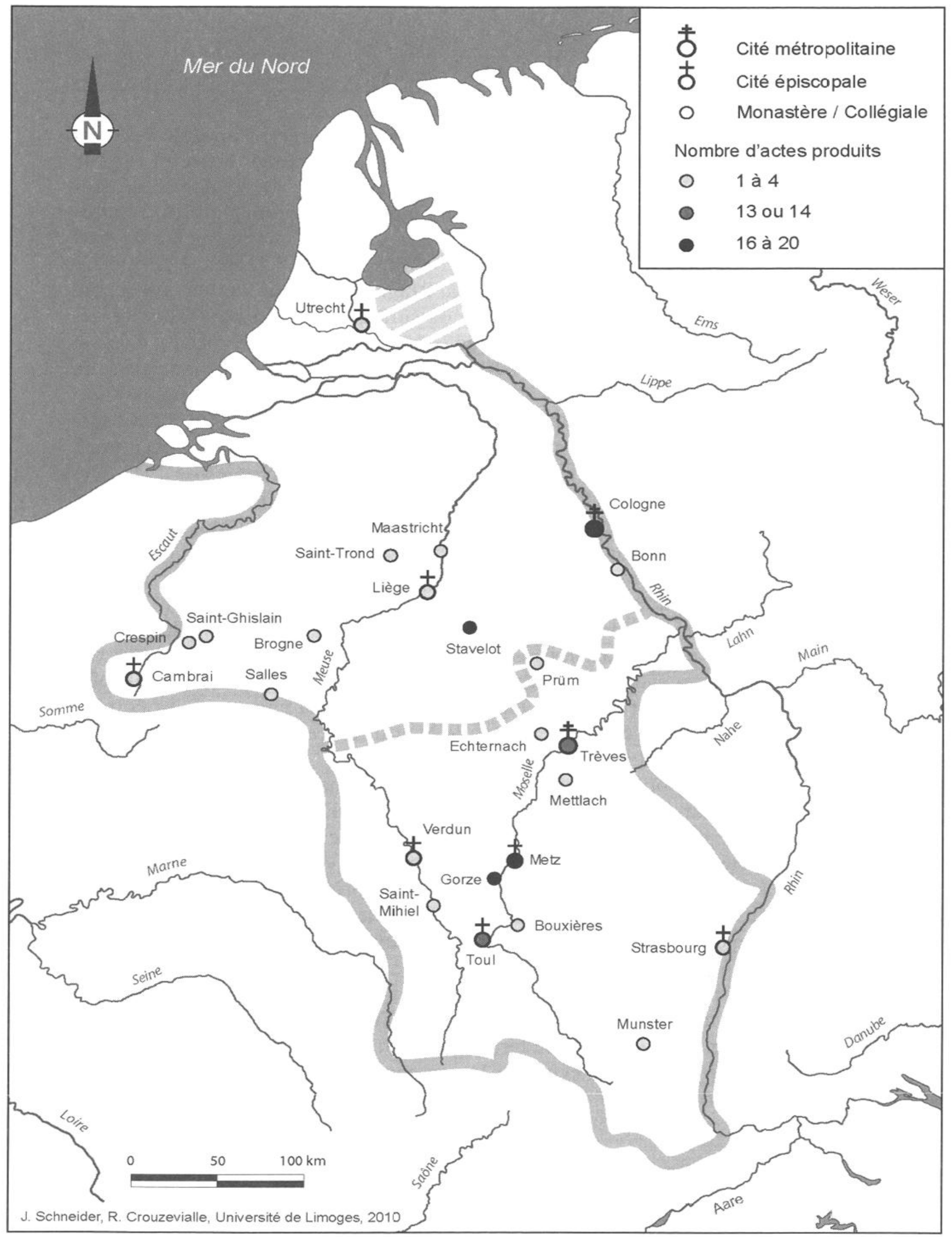

Carte 2: Hiérarchisation des lieux de production d'actes (855-959). 
Pour arriver à une deuxième conclusion on résumera l'analyse des deux ensembles de données soumis ici à l'examen. Le premier est celui des 148 monastères et collégiales de l'espace lotharingien. De l'analyse de leur répartition spatiale et temporelle, on a pu constater dans la future BasseLotharingie un phénomène de rattrapage qui se manifeste de la part des laïcs dans la fondation de 71 communautés religieuses. Les anciens Pays-Bas se distinguent ainsi de la Rhénanie et plus nettement encore de la Haute-Lotharingie qui reste davantage marquée par l'activité des évêques. Le deuxième ensemble, un catalogue de 264 actes privés, a également démontré un clivage relatif entre le Nord et le Sud lotharingien. La carte 2 met en évidence le rôle des anciennes cités épiscopales qui assurent la production diplomatique, particulièrement en Lorraine et au diocèse de Trèves. On peut y voir une politique menée par quelques évêques ${ }^{71}$. Dans le Nord lotharingien, bien avant l'époque de Notger de Liège, les évêques sont remarquablement moins actifs, à l'exception du puissant archevêque de Cologne. En revanche, on trouve ici davantage de fondations et de donations par des familles nobles dont les activités ne passent pas par un membre de la famille devenu évêque. C'est le grand nombre de petites institutions ayant conservé très peu de chartes qui confirme l'image d'une dynamique modeste qui se développe au $\mathrm{x}^{\mathrm{e}}$ siècle dans les anciens Pays-Bas, portée par des réseaux aristocratiques encore au-dessous des comtes et des évêques. On a vu aussi que la part des femmes y semble plus importante. C'est en même temps le moment de la naissance des familles comtales comme celle des comtes de Namur ou ceux de Hollande, fondateurs des monastères d'Egmond et de Tiel $^{72}$.

En ce qui concerne le lieu de rédaction des chartes, ce qui a été dit pour l'époque carolingienne s'applique aussi à la première moitié du $\mathrm{x}^{\mathrm{e}}$ siècle: l'activité diplomatique reste marquée par une "prise de pouvoir» des monastères ${ }^{73}$. Les activités laïques de dotation et de fondation de monastères et collégiales qu'on a pu observer s'expriment dans des chartes qui restent pour la plupart, dans les mots d'Olivier Guyotjeannin, «un produit des écoles et des scriptoria ${ }^{74}$. Il faut néanmoins constater que les choses commencent à changer, d'abord au Sud semble-t-il. Les grands laïcs, s'ils ne sont pas en situation de disposer d'un scriptorium monastique, vont certes continuer à laisser le champ libre aux hommes d'Église ${ }^{75}$, mais la rédaction des chartes ne se fera pas forcément dans un monastère. Bernhard Zeller a mis en évidence la présence de nombreux presbiteri dans les souscriptions des chartes de Saint-Gall, précisant que des mentions

\footnotetext{
${ }^{1}$ Cf. Th. BAUER, Lotharingien als historischer Raum. Raumbildung und Raumbewußtsein im Mittelalter, Culugne, Weimar, Vienne, 1997 (Rheinisches ARCHIv, 136).

${ }^{72}$ Recueil Charles III, éd. LAUER, $\mathrm{n}^{\circ} 121$; MGH DD O.I, $\mathrm{n}^{\circ} 124$ - VAN Viriet, In kringen,

33 Guyotjeannin, Penuria, p. 32.

74 Guyotjeannin, Penuria, p. 32-33.

75 Bolgard, Mise en écriture, p. 17.
} p. 160-161. 
telles que "actum in atrio sancti ..." signalent le travail d'un scribe nonmonastique ${ }^{76}$. Quelques exemples lorrains, toute réserve gardée quant à la distance géographique, montrent des indices comparables en faveur d'une rédaction hors monastère ou collégiale ${ }^{77}$. L'attribution des chanceliers souscripteurs d'un acte privé à un évêque ou un comte est parfois très problématique $^{78}$. Quatre autres exemples semblent cependant témoigner d'une structure administrative destinée à la rédaction d'actes dans l'entourage d'un comte ${ }^{79}$, tandis qu'au Nord les Régnier gèrent leurs affaires en se servant des scriptoria d'Echternach et de Stavelot.

Dans l'espace des anciens Pays-Bas, le premier acte comtal qui ne soit pas le produit d'un scriptorium monastique est peut-être la donation du comte Robert de Namur au monastère de Waulsort, le 2 juin $946^{80}$. La datation Actum Namuco publice, suivie d'une liste de 15 témoins sans aucun membre du monastère, et la souscription d'un Radradus canchellarius semblent indiquer une rédaction par un clerc, peut-être un moine de Waulsort faisant office de chancelier comtal ${ }^{81}$. L'authenticité de la charte, conservée en original dans le chartrier de Waulsort, a été discutée, mais elle ne semble plus remise en cause depuis l'analyse d'Alain Dierkens ${ }^{82}$. Si on a considéré au début de cette contribution les années 950 comme le moment qui referme le siècle lotharingien, la charte de Robert de Namur de 946 présente peut-être un point de départ pour l'histoire des «chancelleries princières $"$.

76 B. Zlelfer, Writing Charters as a Public Activity: The Example of the Carolingian Charters of St Gall, à paraître dans M. Mostert, P. S. BArnwell dir., Medieval Legal Process, Turnhout.

${ }_{77}$ Cartulaire Gorze, éd. D'Herbomez, n' 55 a. 856: Actum in villa cujus vocabulum est Fagido, in atrio S. Gorgonii, publice ... Godefridus presbiter; n 67 a. 874: Actum in atrio Sancti Laurentii ... Anglevertus (sans précision) - Petit Cartulaire, éd. Gaillard, n 62 a. 959: Actum apud Mortismum in mallo publico (pas de scribe mentionné).

78 TOCK, L'acte privé, p. 532; cf. IDEM, Scribes, souscripteurs et témoins dans les actes privés en France (VII' - début XIF' siècle), Turnhout, 2005, p. 286-295.

${ }^{9}$ Cartulaire Gorze. éd. D'Herbomez, no 106: Actum in villa Dexteriaca, in pleno mallo, ... Ego Adelardus, presbiter indignus, ad vicem Nortmanni cancellarii scripsi (en présence du comte); Chronique Saint-Mihiel, éd. LESORT, no 26 (ARTEM 106): Actum publice in villa Nansiide, ... Ego Gennodius presbiter ad vicem Mauromni cancellarii scripsi et subscripsi (en présence du comte); H. Bloch, Die älteren Urkunden des Klosters S. Vanne zu Verdun,

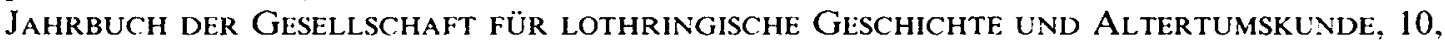
1898, p. 338-449, $\mathrm{n}^{\circ}$ 6: Ego Bertulfus ... cancellarius scripsi rogatus et subscripsi (en présence de trois comtes), no 7: Ego Grimo presbiter et cancellarius scripsi.

80 Chartes Waulsort, éd. Despy, $\mathrm{n}^{\circ} 2$-. D. Misonne, Léloge de Forannan, abbé de Waulsort, dans la charte de Robert de Namur, dans IDEM, Réformes monastiques entre Escaut et Moselle du $x^{\prime}$ au XIr siècle, Maredsous, 2001 (REvle Bénédictine, 111), p. 146-155 (première édition 1966). édition de l'acte p. 148-149.

81 Voir la discussion chez Misonne. L'éloge.

82 Dierkens, Abbayes, p. 164-166 - Pour un pseudo-original du $\mathrm{x}^{\mathrm{c}}$ siècle Misonne. L'éloge. 